\title{
Binding Constraints as Instructions of Binding Machines
}

\author{
António Branco \\ Dept. of Informatics, University of Lisbon \\ Faculdade de Ciências de Lisboa \\ Campo Grande, 1700 Lisboa, Portugal \\ Antonio.Brancodai.fc.ul.pt
}

\begin{abstract}
Binding constraints have resisted to be fully integrated into the course of grammatical processing despite its practical relevance and cross-linguistic generality. The ultimate root for this is to be found in the exponential "overgenerate \& filter" procedure of the mainstream rationale for their satisfaction. In this paper we design an alternative approach based on the view that nominals are binding machines.
\end{abstract}

\section{Introduction}

Binding constraints are an important set of filters in the process of anaphor resolution'. As they delimit the relative positioning of anaphors and their possible antecedents in the grammatical geometry, these constraints are of crucial importance for restricting the search space for antecedent candidates and enhancing the performance of resolvers. From an empirical perspective, they stem from quite robust generalizations and exhibit a universal character. given their parameterized validity across natural languages. From a conceptual point of view, in turn, the relations among binding constraints involve non-trivial symmetry, which lends them a modular nature. Accordingly, they have typically been taken as one of the most intriguing and robust grammar modules.

1 See Annex for examples and the definition of binding constraints.
In contrast to this, however, the formal and computational handling of binding constraints has presented non-negligible resistance when it comes to their integration into the representation and processing of grammatical knowledge.

In its mainstream formulation, the methodology for verifying the compliance of grammatical representations with binding constraints requires a series of extra grammatical parsing steps (Chomsky, 81). More recently, prominent unification-based frameworks either require special purpose extensions of the description formalism for a partial handling of these constraints (LFG: Dalrymple. 93), or offer no integration yet for them into the grammatical setup (HPSG: Pollard and Sag, 94. Backofen et al., 96).

Our primary aim in this paper is to bridge this gap between the grammatical nature of binding constraints and their full integration into grammar processing. In Section 1, we review previous steps towards this goal proposed in the literature. Building on these contributions in Section 2, we introduce the rationale of a new methodology for the verification of binding constraints. In Section 3, in the light of this new approach, we show how these constraints are fully integrated into grammar and the drawbacks of current methodology are overcome.

\section{The Coindexation Paradigm}

The specification of binding constraints have greatly evolved in the last three decades. The device of coindexation for marking anaphoric 
links has, however, remained quite stable. This stems from the fact this device is at the heart of the mainstream methodology for verifying these constraints, a methodology whose basics were proposed in (Chomsky, 80, 81) and have been adopted since then in its different variants.

\subsection{Post-grammatical overgeneration and filtering}

This methodology can be outlined as in Fig. 1 .

After the grammatical parsing of a sentence with $n$ NPs has been completed:

(i) iteration:repeat (ii)-(iii) until all possible different assignments of $n$ indices (tokens) have been exhausted;

(ii) indexation:generate a tree, by assigning indices to its NPs;

(iii) filtering:store the annotated tree if the indexation of NP's respects binding constraints, otherwise delete it.

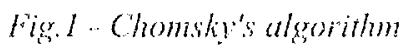

As noted as early as in (Correa. 88), this approach is grossly inefficient. Later fong, 90. showed that its complexity is of exponential order. Moreover. this methodology disregards any concern with interfacing grammar with systems for reference processing. The input for such systems will not be a grammatical representation to be refined vis-à-vis the heuristics for anaphor resolution, but a forest of differently labeled trees that have to be internally searched and compared with each other by allaphor resolvers.

Besides the efficiency issuc, this methodology implies the conceptual awkwardness of having at module of grammar that is not made operative during the grammatical processing, but as an extra-grammatical add-on. Correa, 88, p.123. observed that although the integration of binding constraints "into rules which may be used to derive structure that already satisfies the |constraints| is not a straightforward task". that should be the path to follow. a point also strongly stressed in subsequent elaboration on this issue by Merlo, 93

\subsection{Packaging anaphoric ambiguity}

A first proposal for enhancing integration of binding cosntraints into grammar is due to Correa, 88. Simplifying some details, the proposed algorithm can be outlined as in Fig.2.

Start from the top of the tree with two empty stacks $A$ and $B$ where indices will be collected, respectively local c-commanding indices and non-local c-commanding indices. While walking down a tree where every NP has a distinct index (type):

When an NP is found:

(i) copy: leave a copy of $\mathrm{A}$ (if it is an anaphor) or $B$ (if it is a pronoun) at the NP node;

(ii) assign:talic the first index i of the stack copied into the NP node, take the NP index $j$. and annotate the NP with $\mathrm{j}=\mathrm{i}$;

(iii) collect:add NP index $\mathrm{j}$ to $\mathrm{A}$.

When a local domain border is crossed:

(iv) reset:reset $B$ to AUB.

Fig.2 - Correats algorithm

This algorithm was given two different implementations. one by Correa, 88 . the other by Ingria and Stallard, 89. Further elaboration by Giorgi et al. 90, and Pianesi. 91, led to a restatement of this algorithm using formal language techniques.

The do-it-while-parsing approach of Correats implementation has the advantage of discarding a special-purpose postgrammatical module for binding. That implementation, however. turns out to be dependent on a top-down parsing strategy. On the other hand, Ingria and Stallard's implementation has the advantage of being independent of the parsing strategy adopted. 
This was done however at the cost of still requiring a special purpose postgrammatical parsing module for binding.

Besides the issue of bringing binding into grammar, it is worth noticing that this evolution inside the coindexation paradigm presented a significant improvement and yet a clear drawback. On the one hand, if one disregards step (ii) (a disguised recency heuristic spuriously mixed with binding constraints) and considers the result of verifying binding constraints to be the assignment to an NP of the set of indices of its antecedent candidates, then it is possible to discard the proliferation of indexed trees as a way to express anaphoric ambiguity.

On the other hand, the algorithm is acknowledged not to be able to cope with constraints possibly involving non-local anaphoric links. Principle C, backwards anaphora or cross-over cases were not accounted for (Correa, 88, p.127, Ingria and Stallard, 89, p.268). Moreover, as stack B only contains indices of the non-local c-commanders ${ }^{2}$, but not all indices in the tree except those of the local c-commanders, Principle B is also not correctly accounted for.

\subsection{Packaging non-locality}

Other contributions to improve the coindexation method are due to Dalrymple, 93 and Johnson, 95. Instead of being directed to packaging ambiguity as the one above, they have in common being concerned with packaging non-locality.

\subsubsection{Nodes as mirrors of trees}

Johnson's algorithm is embodied in Prolog code. Abstracting away from details associated to that format, it gets the outline in Fig.3.

\footnotetext{
2 C-command is a configurational version of the command relation where $x$ c-commands $y$ iff the first branching node that dominates $x$ dominates $y$.
}

Although this outline renders the algorithm in a bottom up fashion, Johnson ingeniously developed an implementation that is independent of the parsing strategy resorting to delaying mechanisms. Crucially, in spite of its post grammatical flavor, likewise Correa's implementation, this algorithm does not require postgrammatical processing.

These results were obtained with some

(i) Repeat (ii) until all $\mathrm{NP}_{\mathrm{i}}(1 \leq i \leq n)$ in the tree have been used as starting points;

(ii) Walk up the tree from $\mathrm{NP}_{\mathrm{i}}$ and repeat (iii) until the top node of the tree is reached;

(iii.i) When other locally c-commanding $N P_{j}$ is found:

(iii.i.i) if $\mathrm{NP}_{\mathrm{i}}$ is a short-distance reflexive, annotate $N_{i}$ with $i=j$;

(iii.i.ii) if $\mathrm{NP}_{\mathrm{i}}$ is a non-reflexive, annotate $N P_{i}$ with $i \neq j$;

(iii.ii) When other non-locally c-commanding $\mathrm{NP}_{\mathrm{j}}$ is found: if $\mathrm{NP}_{\mathrm{i}}$ is a non-pronoun, annotate $\mathrm{NP}_{\mathrm{i}}$ with $\mathrm{i} \neq \mathrm{j}$.

Fig.3 - Johnson's algorithm

accessory devices: Each node in the tree is "conceptualized as a pair consisting of a tree and a vertex in that tree" (p.62). Consequently, the whole tree where a given NP appears is locally accessible to be "walked up" since its replica is present at the pair (Category, Tree), which is the NP node itself.

This algorithm improves the coindexation methodology in terms of efficiency as it does not resort to free indexation. Note, however, that the anaphoric ambiguity of pronouns and nonpronouns is not captured (Principles B and C) since grammatical coindexation of pronouns or nonpronouns with their possible antecedents is dismissed. Only reflexives and their antecedents end up coindexed, while the index of a pronoun is only made "unequal" with the 
indices of its (non-grammatical) locally c-commanding antecedents. Nevertheless, even dispensing with free indexation and restricting the representation of anaphoric ambiguity to reflexives, this approach does not get rid of the proliferation of trees: For a given reflexive, each corresponding tree/coindexation represents a different antecedent candidate.

\subsubsection{Equations with regular expressions}

The LFG/Dalrymple, 93, account of binding resorts to a different approach to generalize over the eventual non-locality of anaphoric links. It uses lexical "inside-out equations", a special-purpose extension of the description formalism which may include regular expressions (as in (3) below for long-distance reflexives):

(1) $\mathrm{John}_{i}$ introduced Bill ${ }_{j}$ to himself $\mathrm{F}_{j j}$.

$$
\begin{aligned}
& \text { himself: }\left(\left(\mathrm{OBL} \mathrm{L}_{\text {(ioal }} \uparrow\right) \mathrm{SUBJ}\right)_{\sigma}:=\uparrow_{\sigma} \text { or } \\
&\left(\left(\mathrm{OBL} \mathrm{L}_{\text {Gival }} \uparrow\right) \mathrm{OBJ}\right)_{\sigma}=\uparrow_{\sigma}
\end{aligned}
$$

(2) *John introduced $\mathrm{Bill}_{i}$ to himi

$$
\text { him: } \quad\left(\left(\mathrm{OBL} \mathrm{C}_{\mathrm{G} \text { iat }} \uparrow\right) \mathrm{OBJ}\right)_{\mathrm{Cr}} \neq \uparrow_{\sigma}
$$

(3) Zhangsan yiwei [Lisi, yiwei[ ...ziji i i.j/k/... ...]

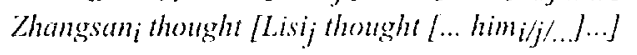

$$
z i j i: \quad((\mathrm{OBJ} * \uparrow) \mathrm{SUBJ})_{\mathrm{S}}=\uparrow_{0}
$$

The right-hand side of the equation stands for the semantic representation $(\sigma)$ of the functional-structure $(\uparrow)$ of the anaphor. The left hand side stands for the semantics of the antecedent: In (3) the Chinese long-distance reflexive is an Object in a functional-structure where one of the upstairs Subjects may be the antecedent.

Although initial scepticism about the tractability of these equations was dissipated by Kaplan and Maxwell, 88, the survey by Backofen et al., 96, reports that no implemented L.FG grammar was known to handle binding. To a significant extent this bears on the fact that many different equations have to be defined for every anaphor:
Each equation specify concrete grammatical functions for the anaphor and its potential antecedent, but either the anaphor or the antecedents may occur with one of a range of several grammatical functions (see a minimal example in (1)). Moreover, it is not defined how non-lexical NPs (e.g. anaphoric definite descriptions, ruled by Principle $C$ ) may be assigned the respective equation.

However these difficulties turn out to be solved, the LFG variant of the coindexation methodology presents the same type of problems of Johnson's approach. The proliferation of representations is not avoided: The ambiguity of reflexives may end up represented by several different grammatical representations. These representations correspond to the satisfaction of the different equations involving different grammatical functions, as in (1), and possibly result also from the several existential interpretations of functional uncertainty in the case of long-distance reflexives, as in (3). Likewise, the ambiguity of pronouns is omitted in the single functional-structure resulting from the universal interpretation of negative equations associated with these anaphoric expressions.

Moreover, the positive equations for reflexives do not require identity of indices between anaphorically related expressions, but instead impose identity of semantic representations, this way incorrectly enforcing any type of anaphora (bound, bridging, e-type, "donkey", etc.) to the sole modility of coreference.

\section{The Concept of Binding Machine}

Being partially successful in overcoming problems of the original post-grammatical "overgenerate \& filter" methodology, each of the contributions mentioned above brought to the fore essential dimensions of binding that have to be concomitantly accounted for. Accordingly, an alternative methodology for binding constraints 
verification should find a way to harmonize all these dimensions: lexicalization, anaphoric ambiguity packaging and non-local context packaging.

Given these hints, a breakthrough depends now on changing some entrenched primitives underlying the conception of binding constraints. These constraints have been basically taken as syntactic well conditions: "[they] capture the distribution of pronouns and reflexives" (Reinhart and Reuland, 93, p.657). In line with Gawron and Peters, 90, however, we take them as conditions on semantic interpretation, as they delimit non-local aspects of meaning composition.

In what follows, we set up a semantics-driven methodology for verifying binding constraints, organized under the rationale that an NP is a binding machine: (i) it reads a representation of the context; (ii) updates its own semantics given this context and its own anaphoric potential (in accordance with its binding constraint, if it is a non-quantificational NP); (iii) and makes a contribution to the context, against which other NPs are interpreted. This rationale is in line with the insights of Johnson and Klein,90 concerning the processing of the semantics of nominals, and also the spirit (but by no means the letter) of the dynamic semantics framework (Chierchia, 95).

The output of a nominal $n$ as a binding machine is simply the incrementing of the context with a copy of its reference marker (Kamp and Reyle, 93). The internal state of the machine after its operation is a compacted representation of the anaphoric potential of $n$, if any, under the form of the set of the reference markers of the grammatically admissible antecedents of $n-$ this internal state results from the binding constraint, lexically associated to $n$, being applied to the input. The input is a representation of the relevant aspects of the context under the form of a set of three lists of reference markers,
$\mathbf{A}, \mathbf{Z}$ and $\mathbf{U}$, from which the internal state/semantics of anaphors can be computed.

Taking $n$ and its subcategorizing predicator $p, A$ is the list with the reference markers of the complements of $p$ ordered according to their relative obliqueness; $Z$ includes the elements of A plus the reference markers of the upstairs predicators directly or indirectly selecting the domain of $p$, observing the multiclausal obliqueness hierarchy; and $\mathbf{U}$ is the list of all reference markers in the discourse context.

Given this setup, the verification of binding constraints consists in a few simple steps. If $n$ is a short-distance reflexive, $\mathrm{A}^{\prime}$ is associated to its semantic representation, where $A^{\prime}$ contains the reference markers of the o-commanders of $n$ in A. If $n$ is a long-distance reflexive, its semantic representation includes $Z^{\prime}$, such that $Z^{\prime}$ contains the o-commanders of $n$ in $\mathbf{Z}$. If $n$ is a pronoun, the set $\mathbf{B}=\mathrm{U} \backslash\left(\mathrm{A}^{\prime} \cup\left\{\mathrm{refm}_{n}\right\}\right)$ is coded into its representation. Finally if $n$ is a nonpronoun, its semantics keeps a copy of $\mathrm{C}=\mathrm{U} \backslash\left(Z^{\prime} \cup\left\{\right.\right.$ refm $\left.\left._{n}\right\}\right)$.

\section{An HPSG exercise}

This methodology can be easily accommodated in a unification-based framework such as HPSG. We designed an extension to the UDRT component for HPSG of Frank and Reyle, 95. This component is encoded as the CONT(ENT) value, which is enhanced now with feature ANAPH(ORA). On a par with this extension, also the NONLOC(AL) value is extended with the new feature BIND(ING), with subfeatures LIST-A, LIST-Z, LIST-U and LIST-protoU.

The SYNSEM value of a pronoun is as follows:

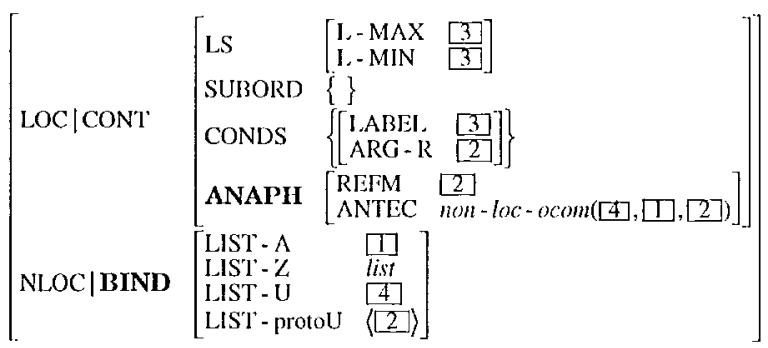


The relational constraint non-loc-ocomm takes (in first argument) all markers in the context. given in LIST-U value, and remove from them both the local o-commanders (included in second argument) of the pronoun and the pronoun itself (in third argument).

Under the conception of nominals as binding machines, LIST-A, LIST-Z and LIST-U stand for the input, ANTEC(EDENTS) encodes the internal state, and REF(ERENCE)M(ARKER) encodes the output. The SYNSEM of other anaphors, ruled by Principles A, C or Z, are quite similar to the one above. The major difference lies in the relational constraints in ANTEC value, which encode the corresponding binding constraint ${ }^{3}$.

Turning now to the lists with reference markers, we handle them by means of a new HPSG principle, the Binding Domains Principle. This principle consists of three clauses constraining signs and their values with respect to these lists. Due to space limitations ${ }^{4}$, we illustrate this Principle with its Clause I, for LIST-U and LIST-protou:

\section{Binding Domains Principle, Clause I}

(i) in every sign, LIST-protou value is identical to the concatenation of LIST-protou values of its daughters;

(ii) in a sign of sort discourse, LIST-protou and LIST-U values are token-identical;

(iii) in a non-NP sign, LIST-U value is token-identical to each LIST-U value of its daughters;

(iv) in an NP sign $k$ :

(iv.i) in Spec-daughter, LIST-U value is the result of removing the elements of LIST-A value of Head-daughter from the LIST-U value of $k$;

\footnotetext{
3 Binding constraints for non-lexical nominals are lexically stated in their determiners.

+ Binding constraints are fully integrated in a computational HPSG grammar, documented in (Branco, 99).
}

(iv.ii) in Head-daughter, LIST-U value is the result of removing the value of $R E F M$ of Spec-daughter from the LIST-U value of $k$.

The HPSG ontology was extended with the sort discourse corresponding to sequences of sentential signs. Subclause (iv) above is meant to avoid what is known as $i$-within-i effect.

\section{Conclusion}

In this paper we designed an alternative to the mainstream postgrammatical "overgenerate \& filter" methodology for the verification of binding constraints. Our semantics-driven methodology is based on the conception of NPs as binding machines. We showed how this innovation helped to integrate binding constraints into grammar representation and processing and to avoid the intractability implied by the mainstream methodology.

\section{Acknowledgements}

I am grateful to Hans Uszkoreit for patient advice and criticism. My thanks go also to Mark Johnson for helpful discussion.

\section{Annex}

Recent results (Xue et al., 94, Branco and Marrafa, 98) indicate that there are four binding constraints:

\section{Principle A:}

A locally o-commanded short-distance reflexive must be locally o-bound.

$$
\text { Lee, thinks [Max; saw himself } *_{i j} \text { l. }
$$

Principle 7:

An o-commanded long-distance reflexive must be o-bound.

$$
\begin{aligned}
& \text { Zhangsani zhidao [Lisij renwei fWangwuk } \\
& \text { zui xihuan ziji/j/kll } \\
& \text { Zhangsan know [Lisi think [Wangwu most like selt]] } \\
& \text { Zhangsani knows that Lisij hinks that Wangwuk likes } \\
& \text { himi/flhimsell }_{k} \text { most (Xue et al., 94) }
\end{aligned}
$$


Principle B:

A pronoun must be locally o-free.

Lee $_{i}$ thinks [ $\operatorname{Max}_{j}$ saw him $\left._{i}{ }_{j}\right]$ ]].

\section{Principle C:}

A nonpronoun must be o-free.

[Kimi's friend $]_{j}$ thinks [Lee saw $\underline{K i m}_{i{ }^{*}}{ }_{j}$.

These constraints are defined on the basis of some auxiliary notions. The notion of local domain involves the partition of sentences and associated grammatical geometry into two zones of greater or less structural proximity with respect to the anaphor. O-command is a partial order under which, in a clause, Subjects o-command Direct Objects, Direct Objects o-command Indirect Objects, and so on, following the usual obliqueness hierarchy of grammatical functions, being that in a multiclausal sentence, the upstairs arguments o-command the embedded arguments, etc. The notion of $o$-binding is such that $x$ o-binds $y$ iff $x$ o-commands $y$ and $x$ and $y$ are coindexed, where coindexation is meant to represent anaphoric links.

\section{References}

Backofen, R., T. Becker, J. Calder, J. Capstick, L. Dini, J. Dörre, G. Erbach, D. Estival, S. Manandhar, A. Mineur, G. van Noord, S. Oepen, H. Uszkoreit. 1996. Final Report of the EAGLES Formalisms Working Group. Luxemburg: EC.

Branco, A., and P. Marrafa. 1998. Long-distance Reflexives and the Binding Square of Opposition. G. Webelhuth, J. Koenig and A, Kathol, eds. Lexical and Constructional Aspects of Linguistic Explanation. Stanford: CSLI Press, 163-177.

Branco, A.. 1999. Reference Processing and its Universal Constraints. Doctoral dissertation. Lisbon: University of Lisbon.

Chierchia, G.. 1995. Dynamics of Meaning. Chicago:Univ. of Chicago Press.

Chomsky, N.. 1980. On Binding. Linguistic Inquiry, $1-46$.
Chomsky, N.. 1981. Lectures on Govermment and Binding. Dordrecht: Foris.

Correa, N.. 1988. A Binding Rule for Government-binding Parsing. COLING'ss, 123-129.

Dalrymple, M.. 1993. The Syntax of Anaphoric Binding. Stanford: CSLI Press.

Erbach, G.. 1995. ProFIT, Prolog with Features, Inheritance and Templates. EACL'95, 180-187.

Fong, S.. 1990. Free Indexation. $A C L^{\prime} 90,105-110$.

Frank, A., and U. Reyle. 1995. Principle-Based Semantics for HPSG. EACL'95, 9-16.

Giorgi, A., F. Pianesi and G. Satta. 1990. A Computational Approach to Binding Theory. COLING'90, 1-6.

Ingria, R., and D. Stallard. 1989. A Computational Mechanism for Pronominal Reference. ACL'89, 262-271.

Johnson, M.. 1995. Constraint-based Natural Language Parsing. Barcelona: 7th ESSLI. Course Notes.

Johnson, M., and E. Klein. 1990. Discourse, Anaphora and Parsing. ACL'90, 197-302.

Kamp, H., and U. Reyle. 1993. From Discourse to Logic. Dordrecht: Kluwer.

Kaplan, R., and J. Maxwell. 1988. An Algorithm for Functional Uncertainty. COLING'88, 297-302.

Merlo, P.. 1993. For an Incremental Computation of Intra-sentential Coreference. IJCAI'93, 1216-1221.

Pianesi. F.. 1991. Indexing and Referential Dependencies within Binding Theory. EACL'93, $39-44$.

Pollard, C., and I. Sag. 1994. Head-Driven Phrase Structure Grammar. Stanford: CSLI Press.

Reinhart, T. and E, Reuland. 1993. Reflexivity. Linguistic Inquiry, 657-720.

Xue, P., C. Pollard, and I. Sig. 1994. A New Perspective on Chinese Ziji. WCCFLI3. Stanford: CSLI Press. 